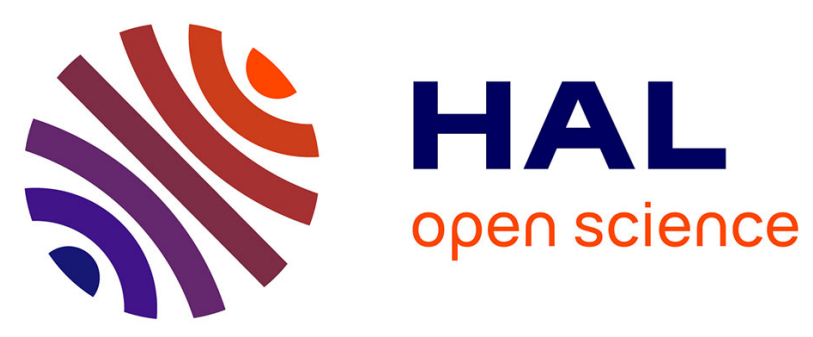

\title{
Stabilization of a Compliant Humanoid Robot Using Only Inertial Measurement Units with a Viscoelastic Reaction Mass Pendulum Model
}

\author{
Alexis Mifsud, Mehdi Benallegue, Florent Lamiraux
}

\section{- To cite this version:}

Alexis Mifsud, Mehdi Benallegue, Florent Lamiraux. Stabilization of a Compliant Humanoid Robot Using Only Inertial Measurement Units with a Viscoelastic Reaction Mass Pendulum Model. IEEE/RSJ International Conference on Intelligent Robots and Systems ( IROS ) 2016, Oct 2016, Daejeon, South Korea. hal-01285643

\section{HAL Id: hal-01285643 \\ https://hal.science/hal-01285643}

Submitted on 9 Mar 2016

HAL is a multi-disciplinary open access archive for the deposit and dissemination of scientific research documents, whether they are published or not. The documents may come from teaching and research institutions in France or abroad, or from public or private research centers.
L'archive ouverte pluridisciplinaire HAL, est destinée au dépôt et à la diffusion de documents scientifiques de niveau recherche, publiés ou non, émanant des établissements d'enseignement et de recherche français ou étrangers, des laboratoires publics ou privés. 


\title{
Stabilization of a Compliant Humanoid Robot Using Only Inertial Measurement Units with a Viscoelastic Reaction Mass Pendulum Model
}

\author{
Alexis Mifsud $^{1,2}$, Mehdi Benallegue ${ }^{1,2}$ and Florent Lamiraux ${ }^{1,2}$
}

\begin{abstract}
To guarantee its balance, a humanoid robot has to respect some contact force constraints. Therefore, traditional controllers generate motions complying with these constraints, but they usually consider the robot as stiff and the joint position perfectly known. However, several robots contain compliant parts in their structure. This flexibility modifies the forces at contacts and endangers balance. However, most solutions to stabilize the robot rely on force sensors. But several humanoid robots aren't equipped with these sensors. This paper has two aims. The first one is to develop a compliance stabilizer using the center of mass position and upper-body orientation through a viscoelastic reaction mass pendulum model. The second objective is to show the performances of such a stabilizer when relying only on an IMU-based state observer. Experimental results on HRP-2 robot show that the stabilization successfully rejects perturbations with high gains using only these IMU signals. Moreover, the actuation of the upper-body orientation provides redundancy, robustness and finally improved performances to the stabilizer.
\end{abstract}

\section{INTRODUCTION}

A humanoid robot has the property to be underactuated. The dynamics of the free-flyer can only be driven by generating reaction contact forces with the environment. At the same time, these forces are constrained by the nature of the contact the robot has with its environment. These constraints are particularly important in the case of upright posture, such like during standing or walking, because contacts only happen with relatively small areas on the ground. Furthermore the height of the center of mass (CoM) reduces the feasible accelerations of the floating base [1]. A common criterion to respect these constraints is to ensure that the position of the Center of Pressure (CoP) also called Zero Moment Point (ZMP) always lies strictly inside the support polygon of the robot [2]. This criterion is derived from the unilaterality of the reactive contact forces, i.e. they must have a positive vertical component.

However, other constraints can apply for contact reaction forces. They can be due for example to geometry, friction cones, underactuation or torque limits. Some constraints may also be the consequence of the presence of compliant parts in the structure of the robot. For instance, the presence of elastic compliance creates a coupling between the reaction forces and its geometric deformation. The reaction forces are

\footnotetext{
*This work is supported by the project ERC Advanced Grant 340050 Actanthrope

*This work was partially funded by the French Romeo-2 project

Alexis Mifsud (alexis.mifsudegmail.com), Mehdi Benallegue (mehdiebenallegue.com) and Florent Lamiraux (florentelaas.fr) are with:

1 CNRS, LAAS, 7 avenue du colonel Roche, F-31400 Toulouse, France

${ }^{2}$ Univ de Toulouse, LAAS, F-31400 Toulouse, France
}

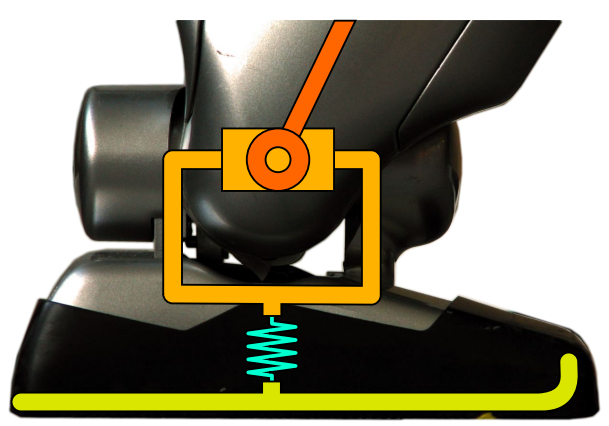

Fig. 1. The foot of HRP-2. Between the ankle joint and the sole of the robot, there is a rubber bush.

then limited by the kinematics constraints and variations of theses forces are bounded by the velocity limits of the robot.

The humanoid robot HRP-2 contains a flexible components between the ankle and the sole [3] (see Fig. 1). This flexibility is used to absorb impacts and protect the force sensors. It is not actuated and its deformations may lead to modifications of the robot dynamics. These dynamics may endanger the balance and lead to a fall. Therefore, the flexibility requires to be precisely estimated and relevantly compensated in order to absorb external perturbations.

In our research, we explore the possibilities of both estimation and control for humanoid and legged robots without using force sensors. In fact, force sensors are usually reliable and play an important role in most stabilization techniques. However, they are usually very expensive, fragile and require fine calibration [4]. Furthermore, some humanoid robots are not equipped with these sensors such as Aldebaran's Romeo [5].

From this viewpoint, we have already shown in previous works that it is possible to design an estimator to reconstruct the flexibility state using only an Inertial Measurement Unit (IMU) and proprioceptive informations provided by the embodied controller [6]. We have shown that using a naive implementation of a CoM controller taking into account the flexibility, we were able to show that this estimation is reliable enough to drive a closed-loop control and stabilization of the center of mass position in the world reference frame [7]. We have also shown that by adding a model of the flexibility dynamics we are able to not only estimate and predict the flexibility state dynamics but also the contact wrenches and thus the ZMP dynamics without using any force sensor [8].

On the basis of the estimation provided by the IMU-based observer, the aim of this paper is to design a novel stabilizer 
for the flexibility able to reject external perturbations. The novelty of this stabilizer is to use both linear and angular momenta as actuation and to rely only on IMU sensors. To do so, the robot is modeled as a rotating mass on top of an inverted pendulum linked to the ground with a compliant joint. We call this model the viscoelastic reaction mass pendulum. As developed in Section V, unlike most current stabilization approaches, which uses these two actuations, they work together in our method in a unified controller and guarantee the stabilization of the flexibility. This stabilizer can also be considered as a validation of the IMU-based estimator, since the angular momentum controller moves the torso of the robot which contains the IMU. The stability of the control is a good assessment that the state observer is able to distinguish between the actuated motion, the deformation of the flexibility and the external perturbations.

In section II we present our control model, to continue in section III by showing how we use this model to construct a stabilizer of the flexibility. We present then in section IV experimental settings and results. And finally we discuss the approach and conclude in section V.

\section{Modeling}

The flexibility of the HRP-2 robot lies between the ankles and the sole of each foot. When subject to a deformation, it creates additional torques on the foot and thus modifies the position of the ZMP. However, in most applications, this robot is modeled and controlled as a perfectly stiff robot [9]. Therefore, a stable posture or a balanced movement for the stiff robot may become unstable with the actual HRP-2 robot. Moreover, external perturbations can induce unpredicted excitations of the flexibility and endanger the balance even more. The flexibility has to be controlled to compensate and reject these disturbances.

To control the flexibility, a model of its dynamics has to be designed. Hereinafter we define the flexibility as in our precedent works [8]. We present then a simplified model of its dynamics using a flexible inverted pendulum with a single rigid-body on top of it. This model is similar as a reaction mass pendulum model[10] but with flexible parts at the base (see Figure 3).

\section{A. Flexibility definition}

HRP-2 is a position-controlled robot with an incompliant actuation. Therefore, apart from the flexibility, we may consider it as a moving but stiff multy-body. We assume that, in the local frame of the robot $\mathcal{R}_{l}$, we perfectly know for each limb $\mathcal{B}_{i}$ its position $\mathbf{c}_{l, i}$ and orientation $R_{l, i}$. We call $\mathcal{R}_{l}$ the "control" frame. We note $M_{l}^{\mathcal{B}_{i}}$ the homogeneous matrix representing the position and orientation of the body $\mathcal{B}_{i}$ in $\mathcal{R}_{l}$ (see Figure 2).

Due to the flexible bush between the ankle and the sole of each foot, we consider that the robot is connected to its environment through a non-stiff contact. Therefore, this flexibility moves the position of each limb to a position $\mathbf{c}_{i}$ and orientation $R_{i}$ in the world reference frame $\mathcal{R}$

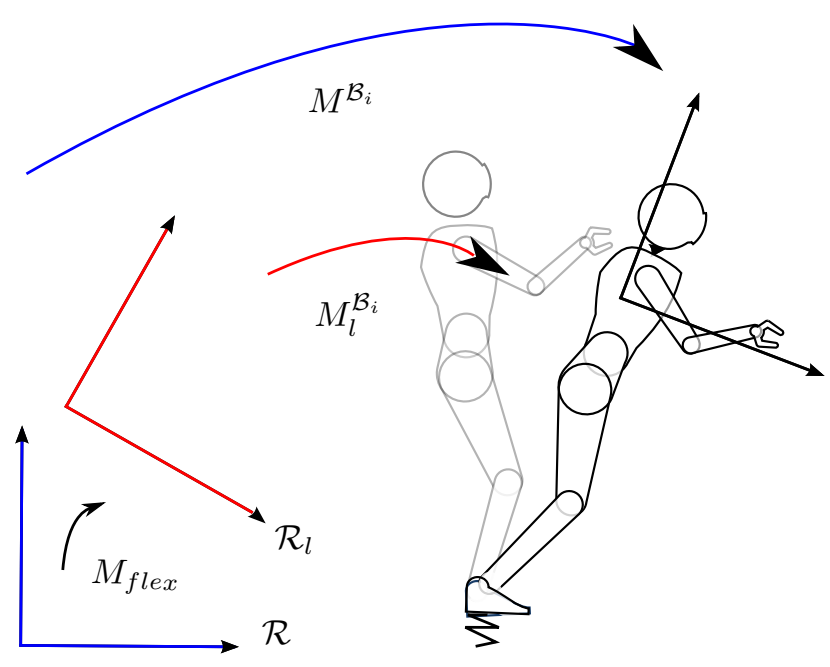

Fig. 2. Definition of the flexibility

represented by the homogeneous matrix $M^{\mathcal{B}_{i}}$ (see Figure 2).

Since the robot is considered as a stiff multi-body, the flexibility modifies the configuration of all the bodies with the same transformation: a rotation of matrix $R$ and a translation of vector $\mathbf{t}$ represented by the homogeneous matrix $M_{\text {flex }}$ such that

$$
M_{\text {flex }}=M^{\mathcal{B}_{i}}\left(M_{l}^{\mathcal{B}_{i}}\right)^{-1}
$$

The flexible bushes are compliant along the 6 degrees of freedom (DoF). In [8], we presented a model of the flexibility which includes them all. Nevertheless, the flexibility is much stiffer in translation and does not require stabilization nor compensation in these DoF. Since this paper deals with stabilization we neglect the translational part $\mathbf{t}$ of this transformation. Hence, we consider that the robot has one contact at the origin, and that the flexibility is only a rotation of matrix $R$. The double support case can be reduced to a single support by using a linearization of the elasticity of translation.

We define then the following flexibility state:

$$
\mathbf{x}_{\text {flex }}=\left(\begin{array}{ll}
\boldsymbol{\Omega}^{T} & \omega^{T}
\end{array}\right)^{T}
$$

where $\boldsymbol{\Omega}$ is defined in such way that $R=\exp \left([\boldsymbol{\Omega}]_{\times}\right)$with $[\cdot]_{\times}$the skew-symmetric operator such that:

$$
\left[\begin{array}{l}
x \\
y \\
z
\end{array}\right]_{\times}=\left[\begin{array}{ccc}
0 & -z & y \\
z & 0 & -x \\
-y & x & 0
\end{array}\right]
$$

and $\omega$ is defined such that $\dot{R}=[\omega]_{\times} R$.

\section{B. Contact torque modeling}

We consider that the robot has one flexible contact with the ground. This flexibility has a viscoelastic response to deformations. In other words, the torque generated at the contact is:

$$
\mathbf{T}_{c}=-K_{T s} \boldsymbol{\Omega}-K_{T d} \omega
$$


where $K_{T s}$ is the elasticity matrix and $K_{T d}$ is the damping matrix. These matrices are both symmetric positive definite.

In the double support case, the flexibility has a different behavior according to the axis of rotation of the deformation. Around the axis which joins the contacts, the behavior is similar as the simple support case, but with summed stiffness and damping. In the other axes the deformation depends on the linear elasticity and the distance between the feet. A linearization of this dynamics can permit to define an equivalent stiffness and damping which reflects these phenomena. This allows to consider the double support case as a single support case with equivalent stiffness and damping matrices.

\section{Newton-Euler dynamics}

The external forces $\mathbf{F}$ are the weight and the contact forces $\mathbf{F}_{c}$. These external forces drive the modification of the linear momentum as expressed in the following Newton's equation:

$$
\mathbf{F}=\mathbf{F}_{c}-g m \mathbf{u}_{z}=\frac{d}{d t}(m \dot{\mathbf{c}})
$$

with $\mathbf{c}$ the center of mass expressed in the world reference frame $\mathcal{R}, \mathrm{m}$ the total mass of the robot and $\mathbf{u}_{z}=\left[\begin{array}{lll}0 & 0 & 1\end{array}\right]^{T}$ is the unit vector along the vertical $\mathbf{z}$ axis.

By expressing this linear momentum in $\mathcal{R}_{l}$, using (1) for the CoM position, and using two time-derivations we obtain:

$$
\begin{aligned}
\mathbf{F}_{c}= & m[\dot{\omega}]_{\times} R \mathbf{c}_{l}+m[\omega]_{\times}^{2} R \mathbf{c}_{l}+2 m[\omega]_{\times} R \dot{\mathbf{c}}_{l} \\
& +m R \ddot{\mathbf{c}}_{l}+g m \mathbf{u}_{z}
\end{aligned}
$$

The external moment $\mathbf{T}$ is composed of the moment of the weight and the contact torque. According to Euler's equation, this external moment is equal to the time-variation of the total angular momentum $\sigma$ of the robot expressed in the world reference frame $\mathcal{R}$. This is summarized in the following equation:

$$
\mathbf{T}=\mathbf{T}_{c}-\left[R \mathbf{c}_{l}\right]_{\times} g m \mathbf{u}_{z}=\dot{\sigma}
$$

If the robot didn't have flexibility, the angular momentum $\sigma$ should have been equal to $\sigma_{l}$, the angular momentum expressed in $\mathcal{R}_{l}$. Because of the flexibility, $\sigma$ can be divided into the angular momentum $\sigma_{l}$ in $\mathcal{R}_{l}$ but expressed in $\mathcal{R}$ and the angular momentum due to the motion of the flexibility. This is summarized in the following expression:

$$
\dot{\sigma}=\frac{d}{d t}\left(R \sigma_{l}+R I_{l} R^{T} \omega\right)
$$

leading to:

$$
\mathbf{T}_{c}=\frac{d}{d t}\left(R \sigma_{l}+R I_{l} R^{T} \omega\right)+\left[R \mathbf{c}_{l}\right]_{\times} g m \mathbf{u}_{z}
$$

\section{The viscoelastic reaction mass pendulum model}

Controlling the contact torques $\mathbf{T}_{c}$ allows to drive the flexibility. Equation (9) shows that for this purpose we need to control the position of the $\mathrm{CoM} \mathbf{c}_{l}$ in $\mathcal{R}_{l}$ and the angular momentum $\sigma_{l}$. More precisely, the angular momentum $\sigma_{l}$ can be rewriten as:

$$
\sigma_{l}=\sigma_{r}+m\left[\mathbf{c}_{l}\right]_{\times} \dot{\mathbf{c}}_{l},
$$

where we can distinguish (i) $\sigma_{r}$ the angular momentum around the CoM, due to the angular velocity of the rigid body in $\mathcal{R}_{l}$ and (ii) the momentum due to its translational velocity in $\mathcal{R}_{l}$. We can also express the inertia matrix $I_{l}$ as:

$$
I_{l}=I_{r}-m\left[\mathbf{c}_{l}\right]_{\times}^{2}
$$

where we can distinguish between (i) $I_{r}$ the inertia tensor of the robot expressed in $R_{r}$, and (ii) the inertia due to the mass $\mathrm{m}$ of the body with a center of mass $\mathbf{c}_{l}$. These two similar decompositions make a convenient grounding for a simplified model of the robot.

It consists in a rotating rigid body of mass $m$, that we call the "trunk", on the top of a massless inverted pendulum. This model is similar to the reaction mass pendulum model [10], except that the contact with the ground is a viscoelastic joint (see Figure 3).

We denote by $R_{l}$ the orientation of the trunk. The tensor of inertia $I_{r}$ is then expressed as:

$$
I_{r}=R_{l} I_{0} R_{l}^{T}
$$

where $I_{0}$ is the inertia tensor of the trunk in its own local frame. By derivation:

$$
\dot{I}_{r}=\left[\omega_{l}\right]_{\times} R_{l} I_{0} R_{l}^{T}-R_{l} I_{0} R_{l}^{T}\left[\omega_{l}\right]_{\times}
$$

where $\omega_{l}$ the angular velocity of the trunk. Similarly we express $\sigma_{r}$ as:

$$
\sigma_{r}=I_{r} \omega_{l}=R_{l} I_{0} R_{l}^{T} \omega_{l}
$$

By derivation we have:

$$
\dot{\sigma}_{r}=\left[\omega_{l}\right]_{\times} R_{l} I_{0} R_{l}^{T} \omega_{l}+R_{l} I_{0} R_{l}^{T} \dot{\omega}_{l}
$$

The dynamics of the flexibility is derived hereinafter. By replacing (10) and (11) in (9) and by identifying $\mathbf{F}_{c}$ using (5) we have:

$$
\mathbf{T}_{c}=\frac{d}{d t}\left(R \sigma_{r}+R I_{r} R^{T} \omega\right)+\left[R \mathbf{c}_{l}\right]_{\times} \mathbf{F}_{c}
$$

Finally, by inverting (16) we are able to have a model of the flexibility dynamics:

$$
\begin{aligned}
\dot{\omega} & =\left(R I_{r} R^{T}-m\left[R \mathbf{c}_{l}\right]_{\times}^{2}\right)^{-1}\left(\mathbf{T}_{c}\right. \\
& -\left[R \mathbf{c}_{l}\right]_{\times}\left(m[\omega]_{\times}^{2} R \mathbf{c}_{l}+2 m[\omega]_{\times} R \dot{\mathbf{c}}_{l}+m R \ddot{\mathbf{c}}_{l}+g m \mathbf{u}_{z}\right) \\
& \left.-\left([\omega]_{\times} R I_{r} R^{T} \omega+R \dot{I}_{r} R^{T} \omega+[\omega]_{\times} R \sigma_{r}+R \dot{\sigma}_{r}\right)\right)
\end{aligned}
$$

Equations (4) and (17), provide us the dynamics at the basis of our stabilization scheme that we describe hereinafter. Note that this dynamics is a contribution of this paper since it is different from the one presented in our previous work [8] since the latter one was more general and does not allow the simplifications provided by the reduced model.

\section{Stabilization}

The model developed in section II allows to predict the flexibility dynamics given the center of mass dynamics in $\mathcal{R}_{l}$, the trunk angular dynamics in $\mathcal{R}_{l}$ and the flexibility state $\mathbf{x}_{\text {flex }}$. In this section we show how we use it in a controller, which aims to stabilize the flexibility around an equilibrium state defined in section III-C with the control architecture presented in section III-B. 


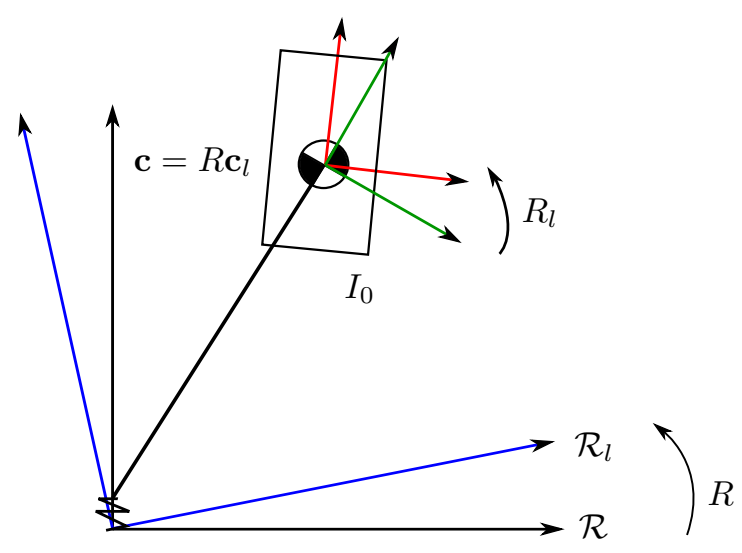

Fig. 3. 2D view of the control model

\section{A. Observation of the flexibility}

The flexibility state is estimated by using a state observer based on an extended Kalman filter presented in [8]. The Kalman filtering technique requires a prediction and an update steps. The prediction is achieved through a model of the flexibility dynamics. This model is very similar to the dynamics of Equation (17), except that it is more complex because we consider explicitly the case of multiple contacts, the translation part is not neglected and the model takes into account all the bodies of the robot. Therefore, this dynamics also depends on the state of the robot expressed in the control frame (e.g. acceleration of the CoM, variations of angular momentum, etc.). These values are available at the control level and are considered well known.

The prediction of the state is corrected using embedded inertial measurement units. These usually include an accelerometer and a gyrometer. These sensors together with the predictor model provide the full observability of the system. We have shown in [8] that besides the accurate observation of the state dynamics, we were able to estimate with relative accuracy the contact forces, without resorting to any force estimator and to reconstruct the trajectory of the ZMP in real-time.

\section{B. Controlling the flexibility}

The stabilization aims at using the CoM positions and trunk orientations, to compensate the disturbances on the flexibility. Even if the flexibility is a 3D rotation, only horizontal axes $\mathbf{x}$ and $\mathbf{y}$ influence balance. In addition, the perturbations faced by an upright standing humanoid robot occur more intensely around the horizontal axes than the vertical. Furthermore, the robot HRP-2 is stiffer around the vertical axis of the foot and does not require stabilization. Therefore, we consider that this part of the flexibility is not relevant in the closed-loop control. Let's define the following state vector:

$$
\mathbf{x}=\left(\begin{array}{llllll}
\mathbf{c}_{l}^{T} & \overline{\boldsymbol{\Omega}}_{l}^{T} & \overline{\boldsymbol{\Omega}}^{T} & \dot{\mathbf{c}}_{l}^{T} & \bar{\omega}_{l}^{T} & \bar{\omega}^{T}
\end{array}\right)^{T}
$$

and the following control vector:

$$
\mathbf{u}=\left(\begin{array}{ll}
\ddot{\mathbf{c}}_{l}^{T} & \overline{\dot{\omega}}_{l}^{T}
\end{array}\right)^{T}
$$

where the barred vector $\bar{*}$ represents the vector $*$ with removed vertical component. The dynamical system

$$
\dot{\mathbf{x}}=f(\mathbf{x}, \mathbf{u})
$$

is the combination of the dynamics of Equations (17), (12), (13), (14) and (15) and the trivial dynamics of $\mathbf{c}_{l}^{T}$ and $\bar{\Omega}_{l}{ }^{T}$. Since the aim of our approach is the stabilization around the desired state, the local dynamics is approximated by its linearization. The desired $x^{*}$ state is described in the next subsection. The linearization and discretization of this dynamics defines the matrices $\mathrm{A}$ and $\mathrm{B}$ such that:

$$
\delta \mathbf{x}_{k+1}=A \delta \mathbf{x}_{k}+B \mathbf{u}_{k}
$$

with $\delta \mathbf{x}_{k}=\mathbf{x}_{k}-\mathbf{x}^{*}$ and $A$ and $B$ are matrices of appropriate dimensions.

To generate the control, we use a Linear Quadratic Regulator (LQR) to minimize over the control space the following quadratic cost $L$ such that:

$$
L=\sum_{k=0}^{N} \mathbf{x}_{k}^{T} Q \mathbf{x}_{k}+\mathbf{u}_{k}^{T} R \mathbf{u}_{k}
$$

where $\mathrm{N}$ is the horizon of the prediction and $\mathrm{Q}$ and $\mathrm{R}$ are respectively weight matrices on the state and the control. Using the model of Equation (21), this problem boils down to solving a Riccati Equation, which provides us with the optimal gain matrix $G$ such that $\mathbf{u}_{k}=G \delta \mathbf{x}_{k}$ induces the minimum cost $L$. The tuning of the cost matrices $Q$ and $R$ allows to modify the responsiveness and the stiffness of the control.

\section{Desired state}

By setting $\dot{\mathrm{x}}=\mathbf{0}$ in (17) we obtain the following equality representing an equilibrium criterion which provide us with all the equilibrium states of the system:

$$
K_{T s} \boldsymbol{\Omega}_{0}=\left[R_{0}\left(\mathbf{c}_{l, 0}\right)\right]_{\times} g m \mathbf{u}_{z}
$$

where $\Omega_{0}$ and $R_{0}$ equilibrium values for the flexibility and $\mathbf{c}_{l, 0}$ an equilibrium value for the position of the center of mass.

To keep the best control on the system, we choose to minimize the energy stored on the flexibility and thus consider the case where the flexibility is at rest. According to Equation (4), this correspond to the null orientation of the flexibility $\boldsymbol{\Omega}_{0}=\mathbf{0}$. The equilibrium criterion of Equation (23) gives naturally that the CoM has to be above the contact point (at the origin) $\mathbf{c}_{l, 0}=\left(\begin{array}{lll}0 & 0 & l\end{array}\right)^{T}$ where $l$ is any height of the CoM. Of course, the orientation of the trunk $\mathcal{R}_{l}$ can be arbitrarily chosen, we set it to identity. This equilibrium point is the point of the linearization of the model in Equation (20).

This equilibrium state is passively stable if and only if the flexibility stiffness is higher than $m g l$. Nevertheless, we have shown in [7] that the state is locally controllable around this state using only $\mathrm{CoM}$ actuation. In this paper we also add the actuation of the trunk orientation, and thus we have redundancy in control and the controllability cannot be altered. Therefore the controllability of our system 
guarantees the stability of the closed-loop system around this state.

\section{EXPERIMENTS}

\section{A. Experimental setting}

The stabilizer of the previous section has been implemented in a $\mathrm{C}++$ real time framework. The humanoid used for our experiments is HRP-2. For the wrench model we use isotropic matrices $K_{T s}=k_{t s} \mathbb{I}_{33}, K_{T d}=k_{t d} \mathbb{I}_{33}$, $K_{F s}=k_{f s} \mathbb{I}_{33}$ and $K_{F d}=k_{f d} \mathbb{I}_{33}$ with the following values: $k_{t s}=400$ n.m.rad ${ }^{-1}, k_{t d}=10$ n.m.s.rad ${ }^{-1}, k_{f s}=40000$ n.m ${ }^{-1}$ and $k_{f d}=600$ n.s.m ${ }^{-1}$. These values are inspired from the identification made for a similar HRP-2 robot [11].

The robot is controlled at $200 \mathrm{~Hz}$. Our control environment is the Stack of Tasks framework [12] which is a task-based hierarchical inverse kinematics solver. The tasks tracked by the solver were set to keep (i) both feet on the ground at $20 \mathrm{~cm}$ distance, (ii) the center of mass above the middle position of the line joining the two supports and (iii) the upper-body to a zero orientation. This position respects the equilibrium state describe in section III-C. The actuation acts then on the position of the center of mass and the orientation of the "trunk". Here the trunk will be reduced to the upperbody only because the legs are constrained to guarantee the support tasks. The upper-body of HRP-2 contains most of the mass and an important part of the moment of inertia. Therefore, even if the influence of the degree of freedom is smaller than the full body, it contributes to the stability and performance of the controller as we show hereinafter.

For testing the performances of the stabilizer we perform an experiment where a reproducible perturbation is given to the robot. A constant $>0.5 \mathrm{Kg}$ mass is attached at the end of a pendulum and released from an height of $1.25 \mathrm{~m}$ to hit the robot horizontally. As stated in [8], HRP-2 is relatively stiff on sideways during double support, so we performed the perturbations in the sagittal plane.

Four different scenarios were compared. The first one is the robot without stabilization. The second one is the robot with the closed-loop stabilizer coupled to the IMU state observer. The third one uses the same gain matrix $G$ as the stabilizer, but with removing all the parts related to the trunk actuation. The fourth setting is to keep only the trunk actuation and to remove the actuation of the CoM. Of course, the gains of the two last settings are not optimal for the criterion (22). The purpose of testing them is to isolate each actuation and watch in detail how it behaves against excitation.

\section{B. Results}

We can see in Figure 4 the ZMP/CoP of the stabilized vs non stabilized robot over time when the perturbation occurs. This CoP is measured using the force sensors of the feet, but they were not used for the closed-loop control. We first see that the CoP is not exactly at zero at the beginning, this is due to biased and errors in the kinematic model. We also see that the stabilization improves significantly the disturbance absorption time of the system. The oscillations are reduced

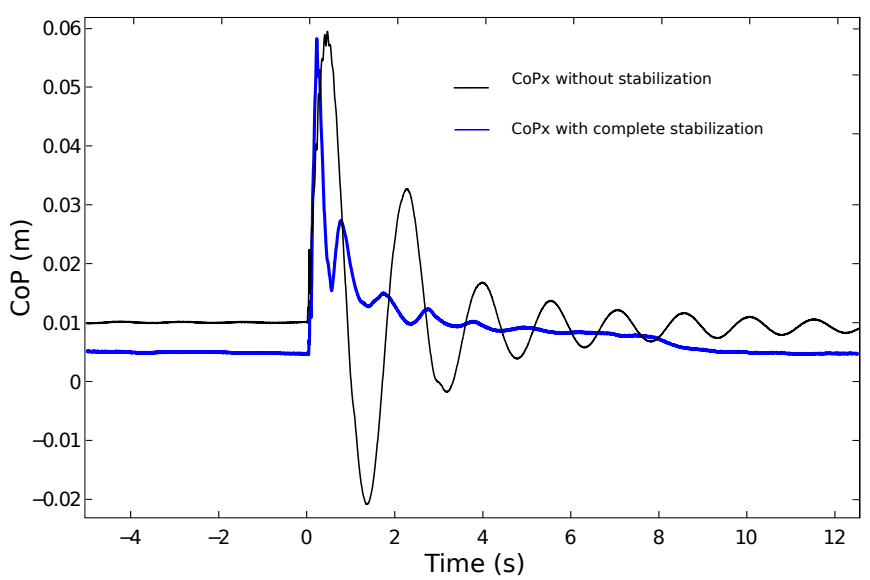

Fig. 4. Response of the system to an impact at $t=0 \mathrm{~s}$ : (1) in bold blue with the stabilizer and (2) in black without the stabilizer.

very quickly and the permanent mode is reached in a very stable way.

In Figure 5 we show the response of the system when we only use the CoM actuation and when we only use the trunk angular actuation. We see first that the response to the perturbations are comparable between the full stabilizer and the CoM-actuated controller. However, we see the presence of auto-oscillations before and after the perturbations. These oscillations are the sign that the controller is reaching its stability limits, i.e. if we increase the gains there is a risk of instability and divergence of the control. This instability is probably due to modeling errors of the flexibility and the dynamics. We can see also that the static error of the CoP is a bit higher with the CoM actuated controller. These differences mean that the trunk actuation enables the stabilizer to improve robustness to modeling errors and to reach higher gains.

It is also interesting to see that even if the trunk actuation alone has weaker impact on the angular momentum than CoM displacements, it still has a visible stabilization effect. Even when the orientations acts alone, the disturbance is absorbed significantly faster than with the rigid robot.

Finally, it is worth to note that HRP-2 has the IMU in the chest. That means that when the robot is rotating the upper-body to stabilize the robot, it could create perturbations on the measurement of the flexibility. The stability of these results are a good assessment that the estimator presented in our previous work [8] is able to distinguish between the desired motion, the motion due to flexibility deformations and the external perturbations.

All these experiments are shown in the attached video.

\section{Discussion AND ConClusion}

We have seen through this paper the development of a stabilizer based on a viscoelastic reaction mass pendulum model. This model provides a simplified dynamics, yet still taking into account the most important features of the motion: the linear and angular momenta. Thanks to this model, we built a dynamical system with two kinds of actuation: 


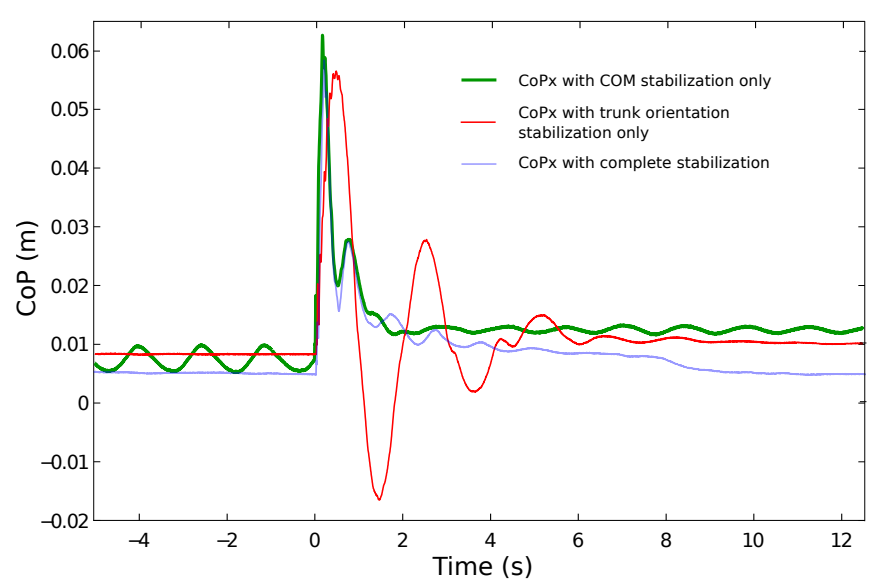

Fig. 5. Response of the system to an impact at $t=0 \mathrm{~s}$ : (1) in bold green using only the CoM actuation, (2) in red with only the trunk actuation and (3) in blurred blue with both the CoM and the trunk actuation.

the position of the center of mass and the orientation of the trunk. The linearization of this dynamical system enables to compute the near optimal gains which drive the unified controller of the flexibility with an optimal quadratic cost.

Several former works also exploited the orientation of the robot for stabilization. This actuation is used to compensate the angular momentum generated by the motion of the center of mass [13], [14]. Nevertheless, this actuation is able to provide more than a simple compensation. It enables redundancy in the control of the angular momentum and increases robustness to external perturbations similarly to what is shown in [15] where a change of variable allows to use the orientation of the torso together with a linear inverted pendulum model. Other interesting results are presented in [16] and [17] where the contact forces are driven to guarantee balance, the tracking of a reference position, and postural control. These works are not designed for robots with passive compliance, but they probably could be adapted to take it into account. However they seem to require accurate force and/or joint torque measurements, which are not necessary with our method.

Our work also constitutes a validation of the state estimator developed in [8]: the estimation is accurate and reactive enough to detect brutal disturbances and drive the stabilization even if the rotations of the trunk influence the signals (the IMU lies in the chest of the robot). Few works, to our knowledge, address the issue of disturbance rejection without the use of force sensors. Most works that don't use force sensors do use joint torque measurement either directly or by using the electric current data [18], [16], [19]. We must cite also the works of running robots which modify the stepping position according to the orientation of the robot [20], [21]. Finally it is worth to note the work by Perrin et al. [22] which uses a clever estimation of the state of the robot using the IMU for the postural control, however they also use force measurements to estimate the deviation of the CoP.

This work is only the first validation of the models we developed. Several extensions have to be achieved to improve the performances. For example, in the case of trajectory tracking, the robot would have to leave the neighborhood of the equilibrium state and the simple linearization would be an insufficient model. In this case a non linear model preview control can be introduced. This model could also takes explicitly into account the constraints related to balance and joint limits. Finally, the introduction of the force sensor would enable us not only to have a more accurate estimation as we have shown in [23], and therefore a more reactive control. This addition should also allow to estimate ground inclinations and external forces and generate more accurate behavior.

\section{REFERENCES}

[1] P.-B. Wieber. On the stability of walking systems. In Proceedings of the International Workshop on Humanoid and Human Friendly Robotics, Tsukuba, Japan, 2002.

[2] M. Vukobratović and B. Borovac. Zero-moment point - thirty five years of its life. International Journal of Humanoid Robotics, 1(01):157-173, 2004.

[3] N. Kanehira, T. Kawasaki, S. Ohta, T. Ismumi, T. Kawada, F. Kanehiro, S. Kajita, and K. Kaneko. Design and experiments of advanced leg module (hrp-2l) for humanoid robot (hrp-2) development. In Intelligent Robots and Systems, volume 3, pages 2455-2460, 2002.

[4] R. M Voyles, J. D. Morrow, and P. K Khosla. The shape from motion approach to rapid and precise force/torque sensor calibration. Journal of dynamic systems, measurement, and control, 119(2):229-235, 1997.

[5] Aldebaran Robotics. Romeo. http://www.projetromeo.com, 2011.

[6] M. Benallegue and F. Lamiraux. Humanoid Flexibility Deformation Can Be Efficiently Estimated Using Only Inertial Measurement Units and Contact Information. In IEEE-RAS International Conference on Humanoid Robots, November 2014.

[7] Mehdi Benallegue and Florent Lamiraux. Estimation and stabilization of humanoid flexibility deformation using only inertial measurement units and contact information. International Journal of Humanoid Robotics, 13(3):1550025-1 to 1550025-20, 2015.

[8] Alexis Mifsud, Mehdi Benallegue, and Florent Lamiraux. Estimation of contact forces and floating base kinematics of a humanoid robot using only inertial measurement units. IROS, September 2015.

[9] K. Kaneko, F. Kanehiro, S. Kajita, K. Yokoyama, K. Akachi, T. Kawasaki, S. Ota, and T. Isozumi. Design of prototype humanoid robotics platform for hrp. In Intelligent Robots and Systems, 2002. IEEE/RSJ International Conference on, volume 3, pages 2431-2436 vol.3, 2002.

[10] Sung-Hee Lee and A. Goswami. Reaction mass pendulum (rmp): An explicit model for centroidal angular momentum of humanoid robots. In Robotics and Automation, 2007 IEEE International Conference on, pages 4667-4672, April 2007.

[11] Y. Mikami, T. Moulard, E. Yoshida, and G. Venture. Identification of hrp-2 foot's dynamics. In Intelligent Robots and Systems (IROS 2014), 2014 IEEE/RSJ International Conference on, pages 927-932, Sept 2014.

[12] N. Mansard, O. Stasse, P. Evrard, and A Kheddar. A versatile generalized inverted kinematics implementation for collaborative working humanoid robots: The stack of tasks. In International Conference on Advanced Robotics, pages 1-6, June 2009.

[13] Zhibin Li, Bram Vanderborght, Nikos G Tsagarakis, Luca Colasanto, and Darwin G Caldwell. Stabilization for the compliant humanoid robot coman exploiting intrinsic and controlled compliance. In Robotics and Automation (ICRA), 2012 IEEE International Conference on, pages 2000-2006. IEEE, 2012.

[14] Shuuji Kajita, Mitsuharu Morisawa, Kanako Miura, Shin'ichiro Nakaoka, Kensuke Harada, Kenji Kaneko, Fumio Kanehiro, and Kazuhito Yokoi. Biped walking stabilization based on linear inverted pendulum tracking. In Intelligent Robots and Systems (IROS), 2010 IEEE/RSJ International Conference on, pages 4489-4496. IEEE, 2010.

[15] Eric C Whitman, Benjamin J Stephens, and Christopher G Atkeson. Torso rotation for push recovery using a simple change of variables. In Humanoid Robots (Humanoids), 2012 12th IEEE-RAS International Conference on, pages 50-56. IEEE, 2012. 
[16] S. Hyon, J.G. Hale, and G. Cheng. Full-body compliant humanhumanoid interaction: Balancing in the presence of unknown external forces. Robotics, IEEE Transactions on, 23(5):884-898, Oct 2007.

[17] C. Ott, M.A. Roa, and G. Hirzinger. Posture and balance control for biped robots based on contact force optimization. In Humanoid Robots (Humanoids), 2011 11th IEEE-RAS International Conference on, pages 26-33, Oct 2011.

[18] Sang-Ho Hyon. Compliant terrain adaptation for biped humanoids without measuring ground surface and contact forces. Robotics, IEEE Transactions on, 25(1):171-178, 2009.

[19] In Hur, Yosuke Matsuki, Nobuyasu Tomokuni, Jian Huang, and Tetsuro Yabuta. Standing stability of surfing robot without force sensor. In the 25th Annual Conference of the Robotics Society of Japan, 3H11, 2007.

[20] Ryosuke Tajima, Daisaku Honda, and K. Suga. Fast running experiments involving a humanoid robot. In Robotics and Automation, 2009. ICRA '09. IEEE International Conference on, pages 1571-1576, May 2009.

[21] B Morris, ER Westervelt, C Chevallereau, G Buche, and JW Grizzle. Achieving bipedal running with rabbit: Six steps toward infinity. In Fast Motions in Biomechanics and Robotics, pages 277-297. Springer, 2006.

[22] N. Perrin, N. Tsagarakis, and D. G. Caldwell. Compliant attitude control and stepping strategy for balance recovery with the humanoid coman. In Intelligent Robots and Systems (IROS), 2013, pages 41454151. IEEE, 2013.

[23] Mehdi Benallegue, Alexis Mifsud, and Florent Lamiraux. Fusion of Force-Torque Sensors, Inertial Measurements Units and Proprioception for a Humanoid Kinematics-Dynamics Observation. In 2015 IEEERAS International Conference on Humanoid Robots, Seoul, South Korea, November 2015. 